\title{
INVARIANT SUBSPACES OF PARABOLIC SELF-MAPS IN THE HARDY SPACE
}

\author{
Alfonso Montes-Rodríguez, Manuel Ponce-Escudero and Stanislav A. \\ SHKARIN
}

\begin{abstract}
We provide a precise description of the lattice of invariant subspaces of composition operators acting on the classical Hardy space, whose inducing symbol is a parabolic non-automorphism. This is achieved with an explicit isomorphism between the Hardy space and the Sobolev Banach algebra $W^{1,2}[0, \infty)$ that induces a bijection between the lattice of the composition operator and the closed ideals of $W^{1,2}[0, \infty)$. In particular, each invariant subspace of parabolic non-automorphism composition operator always consists of the closed span of a set of eigenfunctions. As a consequence, such composition operators have no non-trivial reducing subspaces. For the sake of completeness, we also include a characterization of the closed ideals of the Banach algebra $W^{1,2}[0, \infty)$. Although such a characterization is known, the proof we provide here is somehow different.
\end{abstract}

\section{Introduction}

The problem of giving a precise description of the lattice of invariant subspaces of a bounded linear operator on Hilbert space is one of the most interesting and difficult in operator theory. Very few operators admit a useful description of the lattice of invariant subspaces. In fact, understanding the lattice of a particular operator can solve the invariant subspace problem. This was done by Nordgren, Rosenthal and Wintrobe, [13] and [14]. They consider the composition operator $C_{\varphi}$ acting on the Hardy space, where $\varphi$ is an automorphism of the disk fixing \pm 1 . They show that if every invariant subspace of $C_{\varphi}$ of infinite dimension has a non-trivial invariant subspace, then the general conjecture is true.

Another instance, Beurling's Theorem provides a complete description for the invariant subspaces for the shift operator acting on $\mathcal{H}^{2}$. However, the lattice of invariant subspaces of the shift operator acting on the Bergman space is not completely understood, see [3], [4] or [9, Chapters 7 and 8].

In the present work, we will describe the invariant subspaces of the composition operators $C_{\varphi}$ acting on the Hardy space $\mathcal{H}^{2}$, where $\varphi$ is a parabolic non-automorphism that takes $\mathbb{D}$ into itself, which has the formula

$$
\varphi_{a}(z)=\frac{(2-a) z+a}{-a z+2+a}, \quad \text { where } \Re a>0 .
$$

Received by the editors November 13, 2008.

Key words and phrases. Lattices of invariant subspaces, Hardy spaces, composition operators, Sobolev spaces, Banach Algebras, Gelfand transform.

This work is partially supported by Plan Nacional grant MTM2006-09060 and Junta de Andalucía grants P06-FQM-02225 and FQM-260. 
Since $\varphi_{a}(\mathbb{D})$ is contained in $\mathbb{D}$, Littlewood's Subordination Principle implies the composition operator $\left(C_{\varphi_{a}} f\right)(z)=f\left(\varphi_{a}(z)\right)$ acts boundedly on $\mathcal{H}^{2}$, see the book by Cowen and MacCluer [6] for more details.

If $T$ is an operator on a Hilbert space $\mathcal{H}$ and $x$ is a vector in $\mathcal{H}$, then the smallest invariant subspace of $T$ that contains $x$ is the closure of the linear span of the orbit of $x$ under $T$. If that minimal subspace is $\mathcal{H}$, then $x$ is called a cyclic vector. In the present work we describe all cyclic vectors for $C_{\varphi_{a}}$. In particular, the family of all composition operators induced by parabolic non-automorphism have common dynamics, since they have common cyclic vectors, Corollary 1.2. Moreover, each orbit of any vector under all composition operators induced by parabolic non-automorphisms has a common closure. This is an immediate consequence of Theorem 1.1.

To prove our main result a theorem due to Cowen [5] is essential, see also [6, Theorem 6.1]. He found the spectrum of $C_{\varphi_{a}}$. If $\Re a>0$, the spectrum $\sigma\left(C_{\varphi_{a}}\right)$ is the spiral

$$
\sigma\left(C_{\varphi_{a}}\right)=\{0\} \cup\left\{e^{-a t}: t \in[0, \infty)\right\} .
$$

Indeed, $C_{\varphi_{a}}$ has a well-known family of inner functions as its eigenfunctions,

$$
C_{\varphi_{a}} e_{t}=e^{-a t} e_{t}, \text { where } e_{t}(z)=\exp \left(t \frac{z+1}{z-1}\right) \text { for each } t \geq 0 .
$$

All invariant subspaces we consider in this work will be closed. Let Lat $T$ denote the lattice of invariant subspaces of the bounded linear operator $T$ and let $\mathbb{F}[0, \infty)$ denote the set of closed subsets of $[0, \infty)$. As usual, the closed span of the empty set is the trivial subspace consisting of just the zero vector. We will prove

Theorem 1.1. Let $\varphi$ be a parabolic non-automorphism that takes the unit disk into itself. Then

$$
\text { Lat } C_{\varphi}=\left\{\overline{\operatorname{span}}\left\{e_{t}: t \in F\right\}: F \in \mathbb{F}[0, \infty)\right\} .
$$

In particular, any non-trivial invariant subspace of $C_{\varphi}$ contains a non-trivial eigenfunction of $C_{\varphi}$. As an immediate corollary of the above theorem, we have

Corollary 1.2. Composition operators induced by parabolic non-automorphisms that take the unit disk into itself have the same lattice of invariant subspaces and the same cyclic vectors.

Recall that a subspace that is invariant for an operator as well as for its adjoint is called a reducing subspace. Using Theorem 1.1, we will prove

Theorem 1.3. Let $\varphi$ be a parabolic non-automorphism that takes the unit disk into itself. Then $C_{\varphi}$ has no non-trivial reducing subspace.

The proof of Theorem 1.1 consists of two steps. First, it is shown that the adjoint operator $C_{\varphi}^{\star}$ is similar to the operator of multiplication by a cyclic element in the Sobolev Space $W^{1,2}[0, \infty)$, which is a commutative semisimple regular Banach algebra; the latter idea parallels the one used by Sarason [17] to describe the invariant subspaces of the Volterra operator. Second, the invariant subspaces of such a multiplication operator are precisely the closed ideals of the algebra that can be described by using some elements of the Gelfand Theory. 


\section{An isomorphism from $\mathcal{H}^{2}$ onto the Sobolev space $W^{1,2}[0, \infty)$}

The Sobolev space $W^{1,2}[0, \infty)$ consists of those functions $f$ in $L^{2}[0, \infty)$ absolutely continuous on each bounded subinterval of $[0, \infty)$ and whose derivative belongs to $L^{2}[0, \infty)$. It is well-known and easy to check that the space $W^{1,2}[0, \infty)$ becomes a Hilbert space endowed with the inner product

$$
\langle f, g\rangle_{1,2}=\frac{1}{2} \int_{0}^{\infty}\left(f(t) \overline{g(t)}+f^{\prime}(t) \overline{g^{\prime}(t)}\right) d t .
$$

The corresponding norm will be denoted by $\|\cdot\|_{1,2}$. Similarly, we can define $W^{1,2}(\mathbb{R})$.

We will show up an isomorphism, which is closely related to the eigenfunctions of $C_{\varphi}$, between the Hardy space $\mathcal{H}^{2}$ and the Sobolev space $W^{1,2}[0, \infty)$ that will be crucial to prove Theorem 1.1. The inner functions $e_{t}(z)=\exp (t(z+1) /(z-1))$, with $t \geq 0$, allow us to consider a complex valued function for each $f$ in $\mathcal{H}^{2}$ defined by

$$
(\Phi f)(t)=\left\langle f, e_{t}\right\rangle_{\mathcal{H}^{2}}, \quad t \geq 0 .
$$

The key point to prove that $\Phi$ is an isomorphism from $\mathcal{H}^{2}$ onto $W^{1,2}[0, \infty)$ is to consider the operator $\Psi$ that to each $f$ in $L^{2}(\mathbb{T})$, here $\mathbb{T}$ denotes the unit circle, assigns the function defined as

$$
(\Psi f)(t)=\left\langle f, e_{t}\right\rangle_{L^{2}(\mathbb{T})}, \quad t \in \mathbb{R} .
$$

Let $W_{0}^{1,2}[0, \infty)$ denote the subspace of functions in $W^{1,2}(\mathbb{R})$ that vanish on $(-\infty, 0]$. The space $W_{0}^{1,2}(-\infty, 0]$ is defined similarly. Finally, let $\Pi$ denote the upper half-plane of the complex plane. The Hardy space of the upper half-plane $\mathcal{H}^{2}(\Pi)$ consists of those functions $f$ analytic on $\Pi$ for which the norm

$$
\|f\|_{\mathcal{H}^{2}(\Pi)}^{2}=\sup _{y>0} \int_{-\infty}^{\infty}|f(x+i y)|^{2} d x
$$

is finite, see [16, p. 372]. We will still maintain the symbol $\mathcal{H}^{2}$ for the Hardy space of the unit disk. We have

Theorem 2.1. The map $\Psi$ is an isometric isomorphism from $L^{2}(\mathbb{T})$ onto $W^{1,2}(\mathbb{R})$. In addition, $\Psi\left(z \mathcal{H}^{2}\right)=W_{0}^{1,2}[0, \infty)$ and $\Psi\left(\bar{z} \overline{\mathcal{H}}^{2}\right)=W_{0}^{1,2}(-\infty, 0]$.

Proof. For each $f$ in $L^{2}(\mathbb{T})$, we have

$$
(\Psi f)(t)=\frac{1}{2 \pi} \int_{0}^{2 \pi} f\left(e^{i \theta}\right) \exp \left(t \frac{1+e^{i \theta}}{1-e^{i \theta}}\right) d \theta, \quad t \in \mathbb{R} .
$$

The change of variables $x=i\left(1+e^{i \theta}\right) /\left(1-e^{i \theta}\right)$ yields

$$
(\Psi f)(t)=\frac{1}{\pi} \int_{-\infty}^{\infty} f\left(\frac{x-i}{x+i}\right) \frac{e^{-i t x}}{1+x^{2}} d x, \quad t \in \mathbb{R} .
$$

Therefore, $\Psi=\mathcal{F} M T$, where $\mathcal{F}$ denotes the Fourier transform,

$$
(M g)(y)=\frac{1}{\sqrt{\pi}} \frac{g(y)}{\sqrt{1+y^{2}}} \quad \text { and } \quad(T f)(x)=\frac{1}{\sqrt{\pi}} \frac{1}{\sqrt{1+x^{2}}} f\left(\frac{x-i}{x+i}\right) .
$$

The obvious change of variables shows that $T$ is an isometric isomorphism from $L^{2}(\mathbb{T})$ onto $L^{2}(\mathbb{R})$. In addition, the properties of the Fourier transform along Plancherel's Theorem show that $\mathcal{F} M$ is an isometric isomorphism from $L^{2}(\mathbb{R})$ onto $W^{1,2}(\mathbb{R})$, which proves the first statement of the proposition. 
Now, let $f$ be in $z \mathcal{H}^{2}$, that is, $f(z)=z g(z)$ with $g$ in $\mathcal{H}^{2}$. Using (1), we obtain

$$
(\Psi f)(t)=\frac{1}{\pi} \int_{-\infty}^{\infty} g\left(\frac{x-i}{x+i}\right) \frac{e^{-i t x}}{(x+i)^{2}} d x, \quad \text { for each } t \in \mathbb{R} .
$$

Since the map

$$
h \rightarrow \frac{1}{\sqrt{\pi}(x+i)} h\left(\frac{x-i}{x+i}\right)
$$

is an isometric isomorphism from $\mathcal{H}^{2}$ onto $\mathcal{H}^{2}(\Pi)$, see [10, p. 106], and multiplication by $(w+i)^{-1}$ is bounded on $\mathcal{H}^{2}(\Pi)$, we find that $\Psi f$ is the Fourier transform of a function of $\mathcal{H}^{2}(\Pi)$. Thus, the Paley-Wiener Theorem, see [16, p. 372], shows that $\Psi f$, which is continuous, must vanish on $(-\infty, 0]$ and, therefore, $\Psi\left(z \mathcal{H}^{2}\right) \subset$ $W_{0}^{1,2}[0, \infty)$. Similarly, $\Psi\left(\bar{z} \overline{\mathcal{H}}^{2}\right) \subset W_{0}^{1,2}(-\infty, 0]$. The fact that $\Psi\left(z \mathcal{H}^{2}\right)=W_{0}^{1,2}[0, \infty)$ and $\Psi\left(\bar{z} \overline{\mathcal{H}}^{2}\right)=W_{0}^{1,2}(-\infty, 0]$ follows immediately from the orthogonal decomposition $W^{1,2}(\mathbb{R})=W_{0}^{1,2}(-\infty, 0] \oplus\left[e^{-|t|}\right] \oplus W_{0}^{1,2}[0, \infty)$, which in turns follows, being $\Psi$ an isometric isomorphism, from the orthogonal decomposition $L^{2}(\mathbb{T})=\bar{z} \overline{\mathcal{H}}^{2} \oplus[1] \oplus z \mathcal{H}^{2}$ and the fact that $\Psi 1=e^{-|t|}$, where $[f]$ denotes the one-dimensional linear space spanned by the vector $f$. The proof is complete.

Corollary 2.2. The operator $\Phi$ defines an isomorphism from $\mathcal{H}^{2}$ onto $W^{1,2}[0, \infty)$. Indeed, $\|\Phi f\|_{1,2}^{2}=\|f\|_{\mathcal{H}^{2}}^{2}-|f(0)|^{2} / 2$.

Proof. Upon applying Theorem 2.1, $\Phi$ and $\Psi$ coincide on $z \mathcal{H}^{2}$. Therefore, $\Phi$ defines an isometric isomorphism from $z \mathcal{H}^{2}$ onto $W_{0}^{1,2}[0, \infty)$. Since $e^{-|t|}$ is orthogonal to $W_{0}^{1,2}[0, \infty)$, so is $e^{-t} \chi_{[0, \infty)}$. Thus $W^{1,2}[0, \infty)=\left[e^{-t} \chi_{[0, \infty)}\right] \oplus W_{0}^{1,2}[0, \infty)=$ $\Phi 1 \oplus \Phi\left(z \mathcal{H}^{2}\right)=\Phi\left(\mathcal{H}^{2}\right)$, which proves that $\Phi$ is an isomorphism. The formula for the norm is trivial. The proof is complete.

Remark. In [7, Chaps. IV and V], it is also considered the isomorphism $\Phi$. However, the norm on the space $\Phi\left(\mathcal{H}^{2}\right)$ is defined as $\|\Phi(f)\|=\|f\|_{\mathcal{H}^{2}}$, without identifying $\Phi\left(\mathcal{H}^{2}\right)$ with $W^{1,2}[0, \infty)$, and, consequently, more difficult to handle.

Now, we shall see that the adjoint of composition operators induced by parabolic non-automorphism can be regarded as a multiplication operator on $W^{1,2}[0, \infty)$.

Proposition 2.3. Let $\varphi_{a}$, with $\Re a \geq 0$, be as in (1). Then the adjoint of $C_{\varphi_{a}}$ acting on $\mathcal{H}^{2}$ is similar under $\Phi$ to the multiplication operator $M_{\psi}$, where $\psi(t)=e^{-\bar{a} t}$, acting on $W^{1,2}[0, \infty)$.

Proof. Using the eigenvalue equation (2), for each $f \in \mathcal{H}^{2}$, we have

$$
\left(\Phi C_{\varphi_{a}}^{\star} f\right)(t)=\left\langle C_{\varphi_{a}}^{\star} f, e_{t}\right\rangle_{\mathcal{H}^{2}}=\left\langle f, C_{\varphi_{a}} e_{t}\right\rangle_{\mathcal{H}^{2}}=e^{-\bar{a} t}\left\langle f, e_{t}\right\rangle_{\mathcal{H}^{2}}=e^{-\bar{a} t}(\Phi f)(t),
$$

for each $t \geq 0$. Thus $M_{\psi}=\Phi C_{\varphi_{a}}^{\star} \Phi^{-1}$. The result is proved.

The following proposition is another key point to find the description of the Lattice of $C_{\varphi_{a}}$

Proposition 2.4. The operator $M_{\psi}$, where $\psi(t)=e^{-\bar{a} t}$ and $\Re a>0$, acting on $W^{1,2}[0, \infty)$ is cyclic with cyclic vector $\psi$. 
Proof. Let $k_{\alpha}(z)=(1-\bar{\alpha} z)^{-1}$, where $\alpha=(a-1) /(a+1)$, be the reproducing kernel at $\alpha \in \mathbb{D}$ in the Hardy space $\mathcal{H}^{2}$. Since $\Phi k_{\alpha}=\psi$, by Proposition 2.3, it is enough to show $k_{\alpha}$ is cyclic for $C_{\varphi_{a}}^{\star}$. Suppose that $f$ in $\mathcal{H}^{2}$ is orthogonal to the orbit of $k_{\alpha}$ under $C_{\varphi_{a}}^{\star}$. Then, for each $n \geq 0$, we have

$$
0=\left\langle C_{\varphi_{a}}^{\star n} k_{\alpha}, f\right\rangle_{\mathcal{H}^{2}}=\left\langle k_{\alpha}, C_{\varphi_{a}}^{n} f\right\rangle_{\mathcal{H}^{2}}=\left\langle k_{\alpha}, C_{\varphi_{n a}} f\right\rangle_{\mathcal{H}^{2}}=\left\langle k_{\alpha}, f \circ \varphi_{n a}\right\rangle_{\mathcal{H}^{2}}=f\left(\varphi_{n a}(\bar{\alpha})\right) .
$$

Since $\left\{\varphi_{n a}(\bar{\alpha})\right\}$ is not a Blaschke sequence, the function $f$ is zero and the result follows.

An interesting consequence of Corollary 2.2 is a summability theorem for the Laguerre polynomials. Set $u_{n}(z)=z^{n}$. Then $\widetilde{u}_{n}(t)=\left(\Phi u_{n}\right)(t)=L_{n}^{(-1)}(2 t) e^{-t} \chi_{[0, \infty)}$, where $L_{n}^{(-1)}(t)$ is the Laguerre polynomial of degree $n$ and of index -1 . Indeed, since $\widetilde{u}_{n}=$ $\left\langle z^{n}, e_{t}(z)\right\rangle_{\mathcal{H}^{2}}$ is the $n$-th coefficient of the Taylor series of $e_{t}(z)$, by definition of the Laguerre polynomials see [18, p. 97], we have

$$
e_{t}(z)=e^{-t} \exp \left(-\frac{2 t z}{1-z}\right)=\sum_{n=0}^{\infty} e^{-t} L_{n}^{(-1)}(2 t) z^{n} .
$$

Therefore, it follows immediately

Corollary 2.5. Let $\left\{a_{n}\right\}_{n>0}$ be a sequence of complex numbers. Then the series $\widetilde{f}(t)=\sum_{n=0}^{\infty} a_{n} L_{n}^{(-1)}(2 t) e^{-t} \chi_{[0, \infty)}$ converges in $W^{1,2}[0, \infty)$ if and only if $\left\{a_{n}\right\}$ is in the sequence space $\ell^{2}$. Indeed, $\|\widetilde{f}\|_{1,2}^{2}=-\left|a_{0}\right|^{2} / 2+\left\|\left\{a_{n}\right\}_{n \geq 1}\right\|_{2}^{2}$.

As an application of Corollary 2.5, we show the imbedding theorem for $W^{1,2}[0, \infty)$. This is a well known result, see for instance [2]. However, the proof we provide here is very simple and provides the best constant for the imbedding.

Corollary 2.6. Each $f$ in $W^{1,2}[0, \infty)$ satisfies $\|f\|_{\infty} \leq \sqrt{2}\|f\|_{1,2}$ and $\sqrt{2}$ is the best imbedding constant.

Proof. By Corollary 2.5, we can write $f(t)=\sum_{n=0}^{\infty} a_{n} L_{n}^{(-1)}(2 t) e^{-t}$, where $\left\{a_{n}\right\}$ is in $\ell^{2}$. The Cauchy-Schwarz inequality and Corollary 2.5 , for each $t \geq 0$, yields

$$
|f(t)|=\left|\sum_{n=0}^{\infty} a_{n} L_{n}^{(-1)}(2 t) e^{-t}\right| \leq\|f\|_{1,2}\left(2 e^{-2 t}+\sum_{n=1}^{\infty}\left(L_{n}^{(-1)}(2 t)\right)^{2} e^{-2 t}\right)^{1 / 2} .
$$

Since $\left\|e_{t}\right\|_{\mathcal{H}^{2}}=1$, using (2), one easily checks that the quantity into the brackets above equals to $1+e^{-2 t} \leq 2$ and, therefore, $\|f\|_{\infty} \leq \sqrt{2}\|f\|_{1,2}$. The fact that $\sqrt{2}$ is the best imbedding constant is straightforward.

The following well known result follows immediately from Corollary 2.6.

Proposition 2.7. The space $W^{1,2}[0, \infty)$ with the pointwise multiplication is a Banach algebra without identity.

An element $a$ in a Banach algebra $\mathcal{A}$ is called cyclic, if it is cyclic for the bounded multiplication operator $M_{a}$ that assigns to each $b$ in $\mathcal{A}$ the element $a b$. The following result is folklore for specialists in Banach algebras, although usually it is stated for commutative Banach algebras. We include a proof, since we have not been able to find a precise reference. 
Proposition 2.8. Let $\mathcal{A}$ be a Banach algebra. Then the invariant subspaces of multiplication by a cyclic element are the closed ideals of $\mathcal{A}$.

Proof. First, since $\mathcal{A}$ has a cyclic element, it is commutative. Let $a$ be a cyclic element of $\mathcal{A}$ and let $\mathcal{L}$ be an invariant subspace of $M_{a}$. Clearly,

$$
\mathcal{M}_{\mathcal{L}}=\{b \in \mathcal{A}: b x \in \mathcal{L} \text { for all } x \in \mathcal{L}\}
$$

is a closed subalgebra of $\mathcal{A}$. Since $\mathcal{L}$ is an invariant subspace of $M_{a}$, we find that $a \in \mathcal{M}_{\mathcal{L}}$ and, therefore, $\mathcal{M}_{\mathcal{L}}$ contains the subalgebra generated by a and, being $\mathcal{M}_{\mathcal{L}}$ closed and $a$ cyclic, it follows that $\mathcal{M}_{\mathcal{L}}=\mathcal{A}$. Hence, $\mathcal{L}$ is a left ideal and thus, being $\mathcal{A}$ commutative, an ideal of $\mathcal{A}$. On the other hand, each ideal of $\mathcal{A}$ is invariant with respect to $M_{a}$, which finishes the proof.

\section{Proof of Theorem 1.1}

In view of Proposition 2.8, we need to know a description of the closed ideals of $W^{1,2}[0, \infty)$. Such a description is equivalent to the fact that $W^{1,2}[0, \infty)$ has spectral synthesis, see [8] or [1]. From any of these references, we have,

Theorem 3.1. The closed ideals of $W^{1,2}[0, \infty)$ are

$$
\mathcal{I}_{F}=\left\{f \in W^{1,2}[0, \infty): f \text { vanishes on } F\right\}, \quad \text { where } F \in \mathbb{F}[0, \infty) .
$$

Now, we have all the tools at hand to prove Theorem 1.1.

Proof of Theorem 1.1. By Proposition 2.4, the symbol $\psi$ is a cyclic element of the Banach algebra $W^{1,2}[0, \infty)$. Thus, from Proposition 2.8 and Theorem 3.1 it follows that

$$
\text { Lat } M_{\psi}=\left\{\left\{\widehat{f} \in W^{1,2}[0, \infty): \widehat{f} \text { vanishes on } F\right\} \text {, where } F \in \mathbb{F}[0, \infty)\right\} \text {. }
$$

Since $M_{\psi}=\Phi C_{\varphi}^{\star} \Phi^{-1}$, we have

$$
\text { Lat } C_{\psi}^{\star}=\left\{\left\{f \in \mathcal{H}^{2}:\left\langle f, e_{t}\right\rangle_{\mathcal{H}^{2}}=0 \text { for each } t \in F\right\} \text {, where } F \in \mathbb{F}[0, \infty)\right\} \text {. }
$$

Since Lat $C_{\varphi}$ consists of the orthogonal complements of Lat $C_{\varphi}^{\star}$, the statement of Theorem 1.1 follows immediately.

Now, the proof of Theorem 1.3 follows easily.

Proof of Theorem 1.3. Let $F$ be in $\mathbb{F}[0, \infty)$ such that $N_{F}=\overline{\operatorname{span}}\left\{e_{t}: t \in F\right\}$ is nontrivial. We must show that its orthogonal complement $N_{F}^{\perp}$ is not invariant under $C_{\varphi}$. We need the following formula, which is easily checked

$$
\left\langle e_{t}, e_{s}\right\rangle=e^{-|t-s|}, \quad \text { for each } t, s \geq 0 .
$$

First assume that 0 is not in $F$. Set $t_{0}=\min F$. Since $f_{t_{0}}=1-e^{-t_{0}} e_{t_{0}}$ is orthogonal to $e_{t}$ for each $t \geq t_{0}$, we find that $f_{t_{0}}$ is in $N_{F}^{\perp}$. If $N_{F}^{\perp}$ is invariant under $C_{\varphi}$, then $f_{t_{0}}-C_{\varphi} f_{t_{0}}$ is in $N_{F}^{\perp}$. But $f_{t_{0}}-C_{\varphi} f_{t_{0}}=e^{-t_{0}}\left(1-e^{-a t_{0}}\right) e_{t_{0}}$ is also in $N_{F}$, which means that $f_{t_{0}}-C_{\varphi} f_{t_{0}}=0$. Hence, $f_{t_{0}} \equiv 1$, a contradiction.

Assume now that 0 is in $F$. Let $M_{e_{1}}$ denote the multiplication by $e_{1}$. We have

$$
M_{e_{1}}\left(N_{F}\right)=e_{1} \overline{\operatorname{span}}\left\{e_{t}: t \in F\right\}=\overline{\operatorname{span}}\left\{e_{1+t}: t \in F\right\}=N_{1+F} .
$$

Clearly, $M_{e_{1}}$ is a Hilbert space isometry preserving inner products. Therefore,

$$
M_{e_{1}}\left(N_{F}^{\perp}\right)=M_{e_{1}}\left(N_{F}\right)^{\perp} .
$$


Proceeding by contradiction, assume that $N_{F}^{\perp}$ is also invariant under $C_{\varphi}$. Then

$$
M_{e_{1}}\left(C_{\varphi}\left(N_{F}^{\perp}\right)\right) \subseteq M_{e_{1}}\left(N_{F}^{\perp}\right) .
$$

Since, for $f$ in $\mathcal{H}^{2}$, we have $C_{\varphi}\left(M_{e_{1}} f\right)=C_{\varphi}\left(e_{1} f\right)=e^{-a} e_{1} C_{\varphi} f=e^{-a} M_{e_{1}}\left(C_{\varphi} f\right)$, from the above display, it follows that $C_{\varphi}\left(M_{e_{1}}\left(N_{F}^{\perp}\right)\right)$ is included in $M_{e_{1}}\left(N_{F}^{\perp}\right)$. Therefore, from (2) and (3), we immediately see that $C_{\varphi}\left(N_{1+F}^{\perp}\right) \subseteq N_{1+F}^{\perp}$, which is a contradiction because 0 is not in $1+F$. The proof is complete.

\section{Appendix. The closed ideals of $W^{1,2}[0, \infty)$}

For the sake of completeness, we end by providing a proof of Theorem 3.1, which describes the closed ideals of $W^{1,2}[0, \infty)$.

A character on a Banach algebra $\mathcal{A}$ is a linear functional $\varkappa: \mathcal{A} \rightarrow \mathbb{C}$ such that $\varkappa(a b)=\varkappa(a) \varkappa(b)$ for each $a$ and $b$ in $\mathcal{A}$. We observe that any character on a Banach algebra is continuous [11, p. 201], that is, it belongs to the dual space $\mathcal{A}^{\star}$. The spectrum of $\mathcal{A}$ is the set $\Omega(\mathcal{A})$ of non-zero characters of $\mathcal{A}$ equipped with the weak-star topology. It is well-known that the spectrum of any Banach algebra is a Hausdorff locally compact topological space and it is compact whenever $\mathcal{A}$ has identity [11, p. 205].

We start by determining $\Omega\left(W^{1,2}[0, \infty)\right)$. For each $t \geq 0$, let $\delta_{t}$ denote the reproducing kernel at $t$, that is, $f(t)=\left\langle f, \delta_{t}\right\rangle_{1,2}=\left\langle\Phi^{-1} f, e_{t}\right\rangle_{\mathcal{H}^{2}}$ for each $f \in W^{1,2}[0, \infty)$ and where $\Phi$ is the transform defined in Section 2. Since $W^{1,2}[0, \infty)$ is a Hilbert space, the weak-star topology coincides with the weak topology.

Proposition 4.1. The spectrum of the Banach algebra $W^{1,2}[0, \infty)$ is

$$
\Omega\left(W^{1,2}[0, \infty)\right)=\left\{\delta_{t}: t \geq 0\right\} .
$$

Furthermore, the mapping that to each $t$ assigns $\delta_{t}$ is a homeomorphism from $[0, \infty)$ onto $\Omega\left(W^{1,2}[0, \infty)\right)$.

Proof. Clearly, for each $t \geq 0$, the functional $\delta_{t}$ is a character on $W^{1,2}[0, \infty)$, that is, $\delta_{t}$ is in $\Omega=\Omega\left(W^{1,2}[0, \infty)\right)$. To prove that each character on $W^{1,2}[0, \infty)$ is one of the $\delta_{t}$ 's, we consider the Banach algebra $\mathcal{C}^{1}[0,1]$, with pointwise multiplication, endowed with the norm $\|f\|=\max \left\{\|f\|_{\infty},\left\|f^{\prime}\right\|_{\infty}\right\}$. Consider also its Banach subalgebra $\mathcal{A}_{0}=\{f \in$ $\left.\mathcal{C}^{1}[0,1]: f(1)=0\right\}$. Then, it is easy to check that $(T f)(x)=f(x /(1+x))$ defines a bounded operator from $\mathcal{A}_{0}$ into $W^{1,2}[0, \infty)$, which is also an algebra homomorphism. Now, if $\varkappa$ is a character of $W^{1,2}[0, \infty)$, then it is easy to see that the functional $\tilde{\varkappa}$ on $\mathcal{C}^{1}[0,1]$ defined by $\tilde{\varkappa}(f)=\varkappa(T(f-f(1)))+f(1)$ is also a character. Since the characters of $\mathcal{C}^{1}[0,1]$ are the point evaluations $f \rightarrow f(s)$, with $0 \leq s \leq 1$, see [11, p. 204], there is $0 \leq s \leq 1$ such that $\tilde{\varkappa}(f)=f(s)$ for each $f$ in $\mathcal{C}^{1}[0,1]$. If $s=1$, it follows immediately that $\varkappa(T f)=0$ for each $f$ in $\mathcal{A}_{0}$. Hence $\varkappa$ vanishes on the range of $T$, which is dense because it contains $\mathcal{C}_{c}^{\infty}[0, \infty)$. Therefore, $\varkappa$ is the zero functional. If $s \neq 1$, then set $t=s /(1-s) \geq 0$ and observe that $\varkappa(T f)=(T f)(t)$ for each $f \in \mathcal{A}_{0}$. Hence $\varkappa$ and $\delta_{t}$ coincide on a dense set, which implies that $\varkappa=\delta_{t}$. Thus we have shown that $\Omega=\left\{\delta_{t}: t \geq 0\right\}$.

Next, since each $f$ in $W^{1,2}[0, \infty)$ is continuous, so is the mapping $t \rightarrow \delta_{t}$ from $[0, \infty)$ onto $\Omega$. Since $\left\|\delta_{t}\right\|_{1,2} \leq\left\|\Phi^{-1}\right\|\left\|e_{t}\right\|_{\mathcal{H}^{2}}=\left\|\Phi^{-1}\right\|$, we find that $\Omega$ is norm bounded on the dual space. Since the weak topology of a separable Hilbert space is metrizable on bounded sets, it follows that $\Omega$ is metrizable. Thus, to prove that $t \rightarrow \delta_{t}$ is a homeomorphism, it suffices to show that $t_{n} \rightarrow t_{0}$ whenever $\delta_{t_{n}} \rightarrow \delta_{t_{0}}$. Suppose that 
this is not the case, then there is $\varepsilon>0$ such that $\left|t_{n}-t_{0}\right|>\varepsilon$ for each positive integer $n$. Consider the $W^{1,2}[0, \infty)$-function defined for $t \geq 0$ by

$$
f(t)= \begin{cases}\varepsilon-\left|t_{0}-s\right|, & \text { if }\left|t_{0}-s\right| \leq \varepsilon \\ 0, & \text { otherwise. }\end{cases}
$$

Since $\delta_{t_{n}}(f)=0$ and $\delta_{t_{0}}(f)=\varepsilon$, we find that $\delta_{t_{n}}$ cannot converge to $\delta_{t_{0}}$. Therefore, the mapping $t \rightarrow \delta_{t}$ is a homeomorphism. The result is proved.

Now we turn our attention to the structure of the regular ideals of Banach algebras. An ideal $\mathcal{I}$ of a Banach algebra $\mathcal{A}$ is called regular when the quotient algebra $\mathcal{A} / \mathcal{I}$ has identity. In particular, the kernel of any character is a maximal regular ideal. Therefore, the mapping $\varkappa \mapsto$ ker $\varkappa$ defines a one-to-one correspondence between the spectrum of $\mathcal{A}$ and the set of its maximal regular ideals, which is denoted by $\mathfrak{M}$, see [11, p. 202]. Recall also that a complex algebra is called semisimple if the intersection of all maximal regular ideals, called Jacobson's radical, is zero. Thus a commutative Banach algebra $\mathcal{A}$ is semisimple if and only if the elements of $\Omega(\mathcal{A})$ separate points of $\mathcal{A}$, that is, the intersection of the kernels of the characters is zero.

Given $x \in \mathcal{A}$ and $\mathcal{M} \in \mathfrak{M}$, we denote by $\widehat{x}(\mathcal{M})=x \bmod \mathcal{M}$ the image of $x$ under the multiplicative linear functional corresponding to $\mathcal{M}$. The mapping $x \mapsto \widehat{x}$ is a homomorphism from $\mathcal{A}$ into $C_{0}(\mathfrak{M})$ called Gelfand's transform. The Gelfand transform is one-to-one if and only if $\mathcal{A}$ is semisimple [11, p. 207]. Recall also that a Banach algebra $\mathcal{A}$ is said to be regular whenever for each closed set $F \subseteq \mathfrak{M}$ and each point $\mathcal{M}_{0}$ in $\mathfrak{M}$ such that $\mathcal{M}_{0} \notin F$, there is an element $x$ in $\mathcal{A}$ such that $\widehat{x} \equiv 0$ in $F$ and $\widehat{x}\left(\mathcal{M}_{0}\right) \neq 0$.

The next proposition follows immediately from generals results in the book of Rickart, see [15, p. 91],

Lemma 4.2. Let $\mathcal{A}$ be a semisimple regular commutative Banach algebra. Then the closed ideals of $\mathcal{A}$ are

$$
\mathcal{I}_{F}=\left\{\bigcap_{\varkappa \in F} \operatorname{ker} \varkappa: F \text { is closed in } \Omega(\mathcal{A})\right\}
$$

if and only if for each $x \in \mathcal{A}$ there exists a sequence $\left\{x_{n}\right\}$ tending to $x$ in $\mathcal{A}$ and $\widehat{x}_{n}$ vanishes on a neighborhood $U_{n}$ of $h(x)$ with compact complement.

Thus Theorem 3.1 will be proved once we have shown that $W^{1,2}[0, \infty)$ is under the hypothesis of Lemma 4.2.

Proposition 4.3. The Banach algebra $W^{1,2}[0, \infty)$ is semisimple and regular and the mapping $F \rightarrow \bigcap_{t \in F} \operatorname{ker} \delta_{t}$ is one-to-one from $\mathbb{F}[0, \infty)$ onto the set of closed ideals of $W^{1,2}[0, \infty)$.

Proof. Since the characters $\delta_{t}$ 's separate points, the Banach algebra $W^{1,2}[0, \infty)$ is semisimple. To prove that $W^{1,2}[0, \infty)$ is also regular, we have to show that for each closed $F$ in $\Omega$ and each maximal regular ideal $\mathcal{M} \notin F$ there exists $f$ in $W^{1,2}[0, \infty)$ such that $\widehat{f}=0$ on $F$ and $\widehat{f}(\mathcal{M}) \neq 0$. By Proposition 4.1 this is equivalent to show that for every closed set $F \subseteq[0, \infty)$ and each point $t_{0} \in[0, \infty) \backslash F$ there exists $f$ in $W^{1,2}[0, \infty)$ such that $f$ vanishes on $F$ and $f\left(t_{0}\right) \neq 0$, which is obvious.

It remains to show that the last hypothesis of Lemma 4.2 is also fulfilled. Indeed, the Gelfand transform of a function in $W^{1,2}[0, \infty)$ vanishes on a set in $\Omega$ if and only if the 
function vanishes on its preimage under the homeomorphism furnished by Proposition 4.1. Clearly, for each $f$ in $W^{1,2}[0, \infty)$ there is a sequence $\left\{f_{n}\right\}$ in $\mathcal{C}_{c}^{\infty}[0, \infty)$ converging to $f$ and such that the zero set of each $f_{n}$ contains an open neighborhood $U_{n}$ of the zero set of $f$. Then, by Lemma 4.2, each closed ideal of $W^{1,2}[0, \infty)$ is of the form $\bigcap_{t \in F} \operatorname{ker} \delta_{t}$ for some $F$ in $\mathbb{F}[0, \infty)$, so the mapping $F \rightarrow \bigcap_{t \in F} \operatorname{ker} \delta_{t}$ is onto and since $\bigcap_{t \in F} \operatorname{ker} \delta_{t} \neq$ $\bigcap_{t \in G} \operatorname{ker} \delta_{t}$ whenever $F \neq G$, it is also one-to-one. The result is proved.

\section{Acknowledgements}

The authors would like to thank Patrick Ahern for helpful comments.

\section{References}

[1] D. R. Adams and L. In. Hedberg Function spaces and potential theory. Springer-Verlag, Berlin, 1996.

[2] R. A. Adams Sobolev spaces. Pure and Applied Mathematics, Vol. 65. Academic Press, New York-London, 1975.

[3] A. Aleman, H. Hedenmalm and S. Richter, Recent progress and open problems in the Bergman space. Quadrature domains and their applications, Oper. Theory Adv. Appl. 156 (2005), 27-59.

[4] A. Aleman, S. Richter and C. Sundberg, Beurling's theorem for the Bergman space, Acta Math. 177 (1996), 275-310.

[5] C. C. Cowen, Composition operators on $\mathcal{H}^{2}$, J. Operator Theory 9 (1983), 77-106.

[6] C. C. Cowen and B. D. MacCluer, Composition operators on spaces of analytic functions, Studies in Advanced Mathematics. CRC Press, Boca Raton, 1995.

[7] E. Gallardo-Gutiérrez and A. Montes-Rodríguez, The role of the spectrum in the cyclic behavior of composition operators, Mem. Amer. Math. Soc., vol. 167, 2004.

[8] L. G. Hanin, Closed ideals in algebras of smooth functions, Dissertationes Math. (Rozprawy Mat.) 371 (1998), 1-67.

[9] H. Hedenmalm, B. Korenblum and K. Zhu, Theory of Bergman spaces, Graduate Texts in Mathematics, vol. 199. Springer-Verlag, New York, 2000.

[10] K. Hoffman, Banach spaces of analytic functions, Dover Publications Inc., New York, 1968.

[11] K. Y. Katznelson, An introduction to harmonic analysis, Dover Publications Inc., New York, 1976.

[12] R. Mortini, Cyclic subspaces and eigenvectors of the hyperbolic composition operator, Travaux mathématiques, Fasc. VII, (1995), 69-79, Sém. Math. Luxembourg, Centre Univ. Luxembourg, Luxembourg.

[13] E. A. Nordgren, P. Rosenthal and F. S. Wintrobe, Composition operators and the invariant subspace problem, C. R. Math. Rep. Acad. Sci. Canada 6 (1984), 279-283.

[14] E. A. Nordgren, P. Rosenthal and F. S. Wintrobe Invertible composition operators on $H^{p}$, J. Funct. Anal. 73 (1987), 324-344.

[15] C. E. Rickart, General theory of Banach algebras, The University Series in Higher Mathematics, D. van Nostrand Co., Inc., Princeton, New York, 1960.

[16] W. Rudin, Real and complex analysis, McGraw-Hill, New York, 1987.

[17] D. Sarason A remark on the Volterra operator, J. Math. Anal. Appl. 12 (1965), 244-246.

[18] G. Szegö, Orthogonal polynomials American Mathematical Society Colloquium Publications, vol. 23., New York, 1939.

Departamento de Análisis Matemático, Universidad de Sevilla, Aptdo. 1160, Sevilla 41080, SPAIN

E-mail address: amontes@us.es

Departamento de Análisis Matemático, Universidad de Sevilla, Aptdo. 1160, Sevilla 41080, SPAIN

E-mail address: mpe@us.es

Department of Mathematics, King's College London, Strand, London WC2R 2LS, UK

E-mail address: stanislav.shkarin@kcl.ac.uk 